\title{
Antiplatelet antibody may cause delayed transfusion-related acute lung injury
}

This article was published in the following Dove Press journal:

International Journal of General Medicine

9 September 201 I

Number of times this article has been viewed

\author{
Yoshitaro Torii' \\ Toshiki Shimizu' \\ Takashi Yokoi' \\ Hiroyuki Sugimoto' \\ Yuichi Katashiba' \\ Ryotaro Ozasa' \\ Shinya Fujita' \\ Yasushi Adachi ${ }^{2}$ \\ Masahiko $\mathrm{Maki}^{3}$ \\ Shosaku Nomura' \\ 'The First Department of Internal \\ Medicine, Kansai Medical University, \\ Osaka, ${ }^{2}$ Department of Clinical \\ Pathology, Toyooka Hospital, Hyogo, \\ ${ }^{3}$ First Department of Pathology, \\ Kansai Medical University, \\ Osaka, Japan
}

Correspondence: Shosaku Nomura 10-15, Fumizono-Cho, Moriguchi-City, Osaka 570-8507, Japan

Tel +8I 669939453

Fax $+8 \mid 669921293$

Email nomurash@hirakata.kmu.ac.jp
Abstract: A 61-year-old woman with lung cancer developed delayed transfusion-related acute lung injury (TRALI) syndrome after transfusion of plasma- and leukoreduced red blood cells (RBCs) for gastrointestinal bleeding due to intestinal metastasis. Acute lung injury (ALI) recurred 31 days after the first ALI episode. Both ALI episodes occurred 48 hours after transfusion. Laboratory examinations revealed the presence of various antileukocyte antibodies including antiplatelet antibody in the recipient's serum but not in the donors' serum. The authors speculate that antiplatelet antibodies can have an inhibitory effect in the recipient, which can modulate the bona fide procedure of ALI and lead to a delay in the onset of ALI. This case illustrates the crucial role of a recipient's platelets in the development of TRALI.

Keywords: delayed TRALI syndrome, recurrence, anti-platelet antibody

\section{Introduction}

Transfusion-related acute lung injury (TRALI) is a clinical syndrome associated with blood transfusions and is typically characterized by the sudden onset of dyspnea due to bilateral noncardiogenic pulmonary edema. ${ }^{1}$ Although classic TRALI is defined as acute lung injury (ALI) that occurs within 6 hours following transfusion, late-onset ALI associated with blood transfusion has also been observed clinically. It is proposed that this condition is an independent disease entity termed "delayed TRALI syndrome." A recent laboratory investigation using the two-event animal model proposed that a TRALI event could be halted by the depletion of platelets from the recipient animal, ${ }^{3}$ and in addition, there are a few reports on the participation of platelets in TRALI. ${ }^{4,5}$ However, the clinical evidence that supports this rationale is poorly understood. In this report, a patient who experienced a recurrence of delayed TRALI syndrome is presented. Antiplatelet antibodies were found in the recipient rather than in the donors.

\section{Case report}

The patient was a 61-year-old woman who was diagnosed with advanced nonsmallcell lung cancer with multiple brain metastases. She received systemic chemotherapy (carboplatin + paclitaxel) for lung cancer. However, febrile neutropenia $\left(38.5^{\circ} \mathrm{C}\right.$, neutrophil $753 / \mu \mathrm{L}, \mathrm{C}$-reactive protein $41.50 \mathrm{mg} / \mathrm{dL}$ ) and rapidly progressing anemia $(\mathrm{Hb} 5.2 \mathrm{~g} / \mathrm{dL})$ due to intestinal tract bleeding developed after chemotherapy. She was immediately administered two bags $(600 \mathrm{~mL})$ of plasma- and leukoreduced red blood cells (RBCs) on hospital day 1. No apparent complications were observed 
within 6 hours after transfusion; however, 48 hours after the transfusion, sudden development of dyspnea and rapid progression of hypoxemia were observed. A chest radiograph showed bilateral diffuse pulmonary infiltrates without cardiomegaly (Figure 1A). B-type natriuretic peptide levels were within the normal limits. There was no evidence of microbial, Pneumocystis, tubercular, or fungal infection in the lungs. Methylprednisolone sodium succinate was administered for pulmonary edema, but pulmonary infiltrates and hypoxemia continued to worsen (Figure 1B). On day 23, chest radiography showed a significant improvement in pulmonary shadows (Figure 1C). However, bleeding from the intestinal tract continued, and two additional transfusions, each comprising two bags of RBCs, were required. No adverse events were associated with the two additional transfusions. On day 32, an additional transfusion of two bags of RBCs was performed and on day 34, there was a rapid deterioration of the respiratory condition again. Bilateral pulmonary infiltrates were again observed on a chest radiograph (Figure 1D). The patient died of multiple organ failure the next day. An autopsy was performed, and the pathological findings of the lungs indicated diffuse alveolar damage with hyaline membrane formation (Figure 2A).

To elucidate the contribution of platelets to ALI, we tried an immunohistochemical staining for the platelet using antiCD41 antibody against the pulmonary specimen obtained from the autopsy. The results clearly demonstrated the
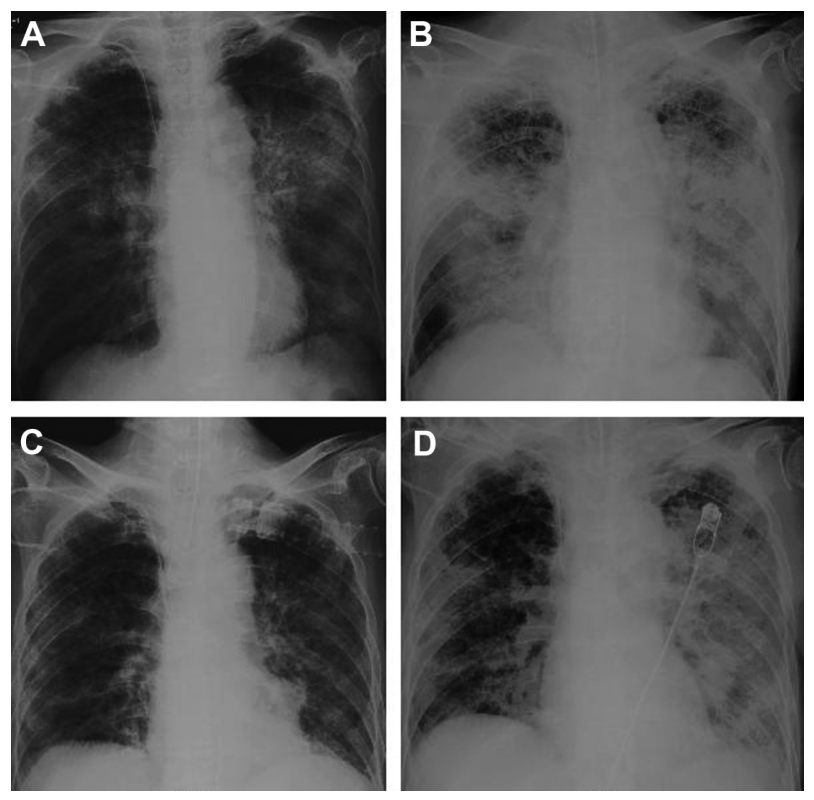

Figure I Chest radiographs. (A) Onset of the first transfusion-related acute lung injury episode on hospital day 3. (B) At the initiation of mechanical ventilation on day 9. (C) A clear chest radiograph on day 23. (D) Onset of the second first transfusion-related acute lung injury episode on hospital day 34 .

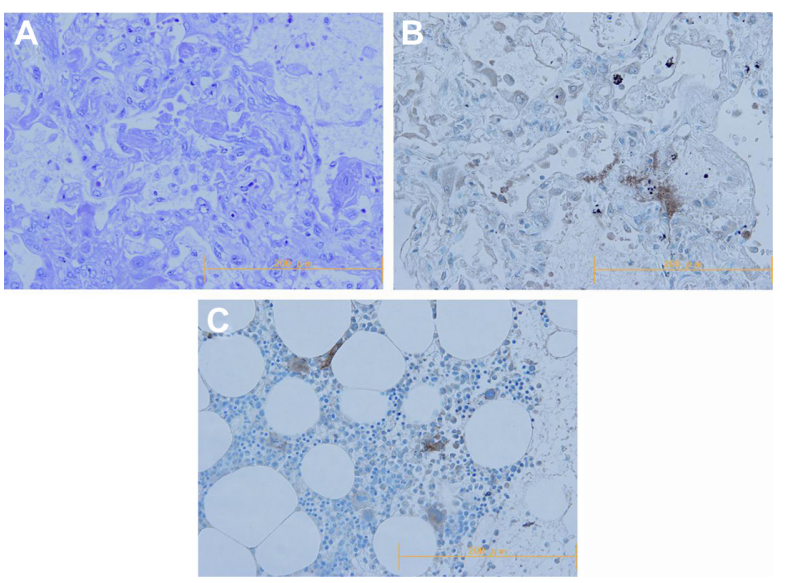

Figure 2 Pathological findings. (A and B) Findings of histological examination of the lung: (A) result of hematoxylin and eosin staining revealed diffuse alveolar damage with hyaline membrane formation $(\times 200)$, (B) result of immunostaining for CD4I demonstrated the sequestration of platelets in the lung $(\times 200)$. (C) Positive control of immunostaining for CD4I (normal bone marrow) $(\times 200)$.

sequestration of platelets in the patient's lung (Figure 2B). These findings strongly suggested the contribution of platelets to the pathogenesis of ALI in this case. Antileukocyte antibodies were not detected in the serum of donors from whom the blood products that led to both of the ALI episodes were obtained. However, antiplatelet Immunoglobulin M antibodies, in addition to the various antileukocyte antibodies (anti-human leukocyte antigen (HLA)-I, anti-HLA-II, antimonocyte, and unclassified antineutrophil antibody), were detected in the serum of the recipient.

\section{Discussion}

Classic TRALI is defined as an ALI that occurs within 6 hours after blood transfusion. ${ }^{6}$ However, this diagnostic criterion for classic TRALI was not met in our case because the two ALI episodes did not occur within 6 hours after the corresponding transfusions. A recent report on the two-event animal model revealed that the environmental status of the recipient can affect the procedure in TRALI. ${ }^{3}$ Looney et al described that platelets in the circulation of the recipient are essential for developing ALI and act as terminal effector cells. ${ }^{3}$ The pathological findings also showed marked sequestration of platelets in the lung in which ALI developed. However, some antiplatelet antibodies have been shown to directly activate platelets. ${ }^{7,8}$

Platelets have been shown to contain members of the chemokine family of the $\mathrm{C}-\mathrm{C}$ class, such as regulated on activation, normal T-cell expressed, and secreted and monocyte chemotactic peptide-3, as well as the CXC class, such as interleukin-8 and epithelial neutrophil-activating peptide-78. ${ }^{9}$ Moreover, recent studies have shown that human platelets 


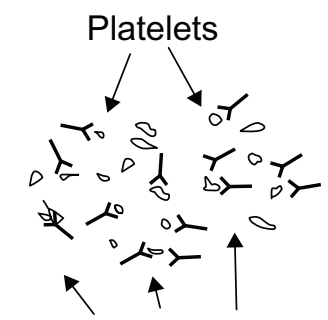

Antiplatelet antibody

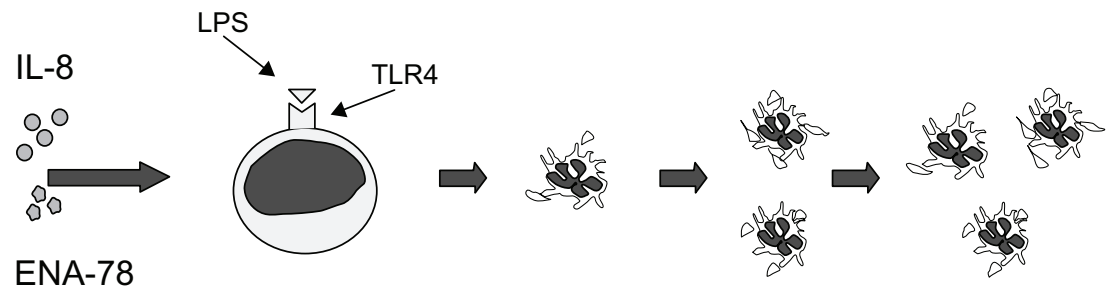

LPS-primed neutrophil

Activation and alveolar recruitment

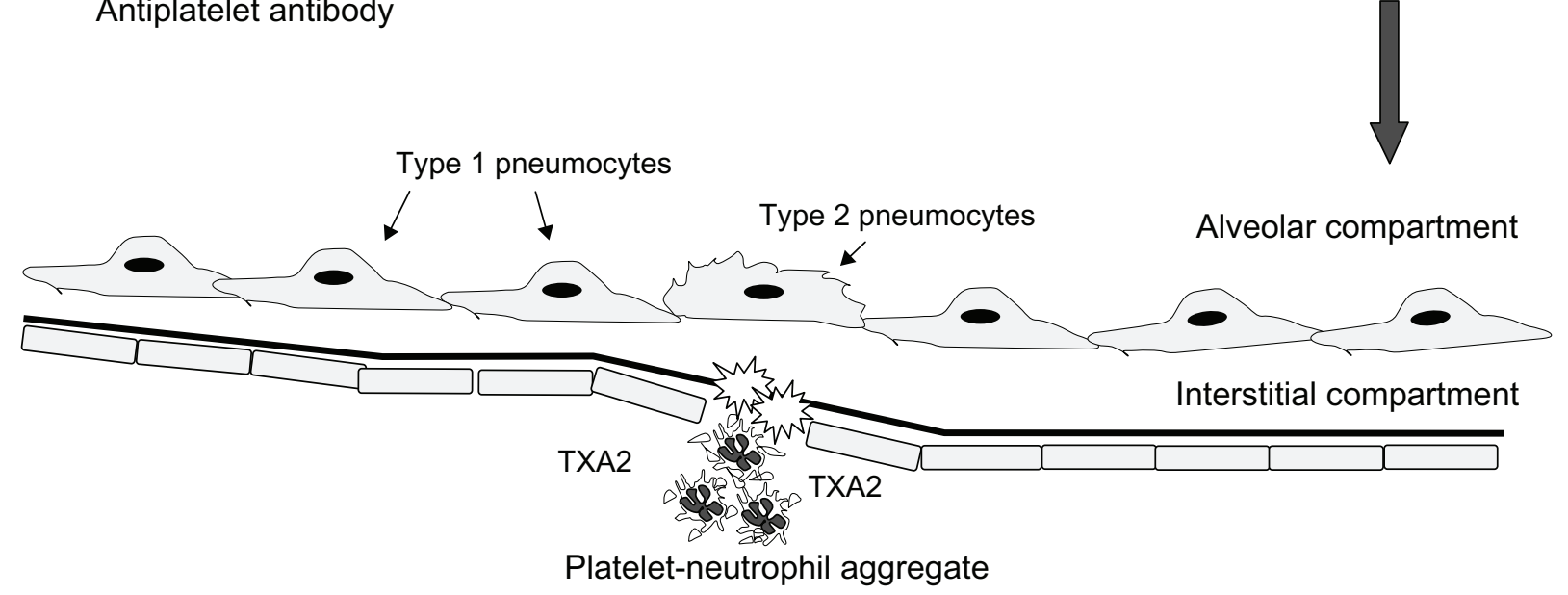

Figure 3 Rationale of delayed transfusion-related acute lung injury. In delayed TRALI, antiplatelet autoantibodies interfere with the release of IL-8 and ENA-78, and plateletneutrophil aggregation and secretion of TXA2, resulting in a delay of onset of acute lung injury.

Abbreviations: ENA-78, epithelial neutrophil-activating peptide (ENA)-78; IL-8, interleukin (IL)-8; LPS, lipopolysaccharide; TRALI, transfusion-related acute lung injury; TRL-4, toll-like receptor 4; TXA2, thromboxane A2.

express Toll-like receptor $4^{10}$ and can sequestrate in the lung in response to lipopolysaccharide (LPS) in a neutrophildependent manner. ${ }^{11,12}$ Taken together, these findings suggest that circulating platelets contribute to the development of TRALI throughout the whole process. In the case documented here, antileukocyte antibodies were not found in the donors, but antiplatelet antibodies were observed in serum from the recipient. It is hypothesized that severe bacterial infection associated with systemic chemotherapy plays a role in LPSpriming, and the transfusion of stored RBCs, which can contain any biological response modifier (eg, lysophosphatidylcholine, arachidonic acid, or hydroxyeicosatetraenoic acid), acts as a "second hit" initiating TRALI. ${ }^{13}$ However, antiplatelet antibodies in the recipient might inhibit intra-alveolar platelet recruitment, resulting in a delay of platelet sequestration. Consequently, it is considered that this delay of platelet sequestration due to antiplatelet antibodies could cause a 48-hour eclipse time from the corresponding blood transfusion to the development of ALI. The present case report suggests an important role for the recipient's platelets and antiplatelet autoantibodies in the development of TRALI (Figure 3). These clinical findings could represent modulation of a TRALI event by environmental factors in the recipient, rather than in the donors.

\section{Disclosure}

The authors declare no conflicts of interest in relation to this work.

\section{References}

1. Popovsky MA, Moore SB. Diagnostic and pathogenetic considerations in transfusion-related acute lung injury. Transfusion. 1985;25: 573-577.

2. Marik PE, Corwin HL. Acute lung injury following blood transfusion: expanding the definition. Crit Care Med. 2008;36:3080-3084.

3. Looney MR, Nguyen JX, Hu Y, Van Ziffle JA, Lowell CA, Matthay MA. Platelet depletion and aspirin treatment protect mice in a two-event model of transfusion-related acute lung injury. J Clin Invest. 2009;119: 3450-3461.

4. Khan SY, Kelher MR, Heal JM, et al. Soluble CD40 ligand accumulates in stored blood components, primes neutrophils through CD40, and is a potential cofactor in the development of transfusion-related acute lung injury. Blood. 2006;108:2455-2462.

5. Tuinman PR, Gerards MC, Jongsma G, Viaar AP, Boon L, Juffermans NP. Lack of evidence of CD40 ligand involvement in transfusion-related acute lung injury. Clin Exp Immunol. 2011;165:278-284.

6. Holness L, Knippen MA, Simmons L, Lachenbruch PA. Fatalities caused by TRALI. Transfus Med Rev. 2004;18:184-188.

7. Nomura S, Yamaguchi K, Kido H, et al. New monoclonal anti-human Fc gamma receptor II antibodies induce platelet aggregation. Clin Exp Immunol. 1991;86:179-184.

8. Yanabu M, Nomura S, Fukuroi T, et al. Platelet activation induced by an antiplatelet autoantibody against CD9 antigen and its inhibition by another autoantibody in immune thrombocytopenic purpura. $\mathrm{Br} \mathrm{J}$ Haematol. 1993;84:694-701.

9. Nomura S, Inami N. Roles of platelet-derived chemokines in various clinical settings. In: Grinwald LR, editor. Chemokine Research Trends. New York: Nova Science; 2007:159-169. 
10. Andonegui G, Kerfoot SM, McNagny K, Ebbert KV, Patel KD, Kubes P. Platelets express functional Toll-like receptor-4. Blood. 2005; 106: 2417-2423.

11. Zhang G, Han J, Welch EJ, et al. Lipopolysaccharide stimulates platelet secretion and potentiates platelet aggregation via TLR4/MyD88 and the cGMP-dependent protein kinase pathway. J Immunol. 2009;182: 7997-8004.
12. Nomura S, Ozaki Y, Ikeda Y. Function and role of microparticles in various clinical settings. Thromb Res. 2008;123:8-23.

13. Silliman CC, Moore EE, Kelher MR, et al. Identification of lipids that accumulate during the routine storage of prestorage leukoreduced red blood cells and cause acute lung injury. Transfusion. 2011. [Epub ahead of print.]

\section{Publish your work in this journal}

The International Journal of General Medicine is an international, peer-reviewed open-access journal that focuses on general and internal medicine, pathogenesis, epidemiology, diagnosis, monitoring and treatment protocols. The journal is characterized by the rapid reporting of reviews, original research and clinical studies across all disease areas.
A key focus is the elucidation of disease processes and management protocols resulting in improved outcomes for the patient. The manuscript management system is completely online and includes a very quick and fair peer-review system. Visit http://www.dovepress.com/ testimonials.php to read real quotes from published authors.

Submit your manuscript here: http://www.dovepress.com/international-journal-of-general-medicine-journal 